www. revis tad y o. com

\title{
Producción y demanda de biodiesel en Brasil: Revisión
}

\author{
Production and biodiesel demand in Brazil: Review
}

\begin{abstract}
Antonio Luiz Fantinel; Sérgio Luiz Jahn y Yesica Ramirez Flores
Universidade Federal de Santa Maria.
\end{abstract}

toni.agronegocio.ufsm@gmail.com; jahn@smail.ufsm.br; yeyiramiflo@hotmail.com

Fecha de recepción: 16-04-2015

Fecha de aceptación: 21-10-2015

\begin{abstract}
Resumen: La producción de biodiesel está aumentando en todo el mundo, emergiendo como una de las principales opciones para resolver los problemas ambientales (uso de combustibles fósiles), problemas financieros (importaciones de combustibles). Este estudio tiene como objetivo caracterizar y analizar la situación actual de la producción brasileña de biodiesel. Por lo tanto, hubo una búsqueda bibliográfica de artículos, boletines y directorios a saber: materias primas, proceso de producción, la producción y la demanda interna, y, finalmente, los costos de producción. A pesar de tener una producción récord en 2014 con 3.072 millones de litros de biodiesel, un $17 \%$ más que el año anterior, existen numerosas barreras relativas a la demanda de biodiesel debido a los altos costos de producción, sobre todo de las materias primas utilizadas para la producción. Sin embargo, cuando se utiliza residuos como el aceite de pollo y sebo de vacuno, su costo se reduce drásticamente en comparación con el uso de aceites vegetales puros.
\end{abstract}

Palabras clave: Biomasa, biodiesel, producción, demanda, Brasil.

Abstract: The production of biodiesel is increasing worldwide, emerging as one of the main options in order to solve environmental problems (use of fossil fuels), financial problems (fuel imports). This study aims to characterize and analyze the current situation of the Brazilian production of biodiesel. Therefore, it is bibliographical research in articles, newsletters and directories to know: raw materials, production process, production and domestic demand, and finally costs of production. Despite having record production in 2014 with 3,072 billion liters of biodiesel, 17\% higher than the previous year, there are numerous obstacles, for the production and biodiesel demand due to high production costs, especially of raw materials used for production. However, when used for the production waste as chicken and beef tallow oil, its cost is dramatically reduced compared to the use of pure vegetable oils.

Keywords: Biomass, biodiesel, broduction, demand, Brazil.

\section{Introducción}

En los últimos años, el mundo se ha enfrentado nuevamente una crisis energética constante, causada por la disminución de los recursos fósiles, las limitaciones ambientales y el aumento de los precios del petróleo (Coronado et al., 2009; Singh y Singh 2010).

En Brasil, como en otros países, a causa de estas situaciones antes mencionadas, están surgiendo nuevas fuentes de energía, clasificadas como los biocombustibles o combustibles renovables, con la intención de sustituir el uso del petróleo y sus derivados como fuente de energía. Entre las diversas fuentes de energía limpia, se puede citar el uso de la biomasa, que está ganando importancia en la matriz energética de muchos países (Matsumoto et al., 2009; Tsai, 2009; Becker et al., 2011).

Los biocombustibles son fuentes de energía obtenidos mediante cualquier biomasa de las plantas que se pueden convertir en energía mecánica, térmica o eléctrica (Mello, 2001; Aneel, 2008). Tienen características físicas similares a los combustibles minerales, pero se diferencian porque son producidos a partir de biomasa procesada químicamente, que se describe como carbono neutral (Hammond et al., 2008).Su combustión no aumenta los niveles de $\mathrm{CO} 2$ en la atmósfera y se obtienen a partir de caña, maíz, semillas oleoginosas y residuos agrícolas, entre otras fuentes (Lima, 2005; Vieira, 2014). 
Permitiendo establecer un ciclo de carbono cerrado, y (CO2) absorbido en crecimiento de la planta y liberado en la combustión del motor cuando el biocombustible se quema, por lo tanto tiene un valor nulo de balance de masa, es decir, su combustión no aumenta la cantidad de $\mathrm{CO} 2$ en la atmósfera, no empeora el efecto invernadero (Santos, 2010; Ferreira et al., 2014).

Entre las diversas fuentes renovables derivados de la biomasa, biodiesel (B100), aparece en el crecimiento de todo el mundo, surge como una de las principales opciones para minimizar los problemas ambientales causados por el uso de combustibles fósiles y los problemas financieros de la país (Foschiera, 2008). Así, el presente estudio tuvo como objetivo caracterizar y analizar la situación actual de la producción y la demanda brasileña de biodiesel.

\section{Metodología}

La investigación se define por Gil (1994), como un proceso formal y sistemático de desarrollo del método científico. El objetivo fundamental de la investigación es encontrar respuestas al problema mediante el uso de procedimientos científicos. Para Minayo (2003), la búsqueda es un factor de oficio, sin renunciar a la creatividad y realizar básicamente un lenguaje incorporado en conceptos, propuestas, métodos y técnicas, este lenguaje se construye con un ritmo particular y propio.

La investigación en cuestión presenta un enfoque cualitativo y cuantitativo. La elección de este tipo de investigación se justifica por el hecho de que se centra en el estudio de un fenómeno social contemporáneo poco investigado y requiere una comprensión más profunda, lo que permite la identificación de los aspectos que caracterizan y hacen más explícito y accesible para la comunidad científica y la sociedad en su conjunto.

Según Martins (2004, p. 292) "la realización de un examen intensivo de los datos, tanto en amplitud como en profundidad, los métodos cualitativos tratan unidades sociales investigados como totalidades."Los instrumentos de recolección de datos utilizados fueron la literatura, y se utilizan artículos, boletines y directorios, de enfoque y análisis de las siguientes variables: materias primas, la producción nacional, la demanda y los costos de producción.

\section{Revisión de literatura}

\subsection{Biodiesel (B100)}

El uso de aceite vegetal para la generación de energía tiene como fecha inicial el año 1895 cuando el Dr. Rudolf Diesel desarrolló el primer motor encendido a la compresión, para la ocasión, a petición de Francia fue utilizado como combustible aceite de maní. Sin embargo, debido a la disponibilidad y el aceite mineral de bajo coste en ese momento, el uso de aceites vegetales no se ha convertido en atractivo con lo que se descartó como fuente de combustible (Puppán, 2002).

En los últimos años, debido a la escasez de petróleo y la amenaza a la calidad de vida de la humanidad, se ha a profundado debido a las emisiones de gases de efecto invernadero, tiene sea profundado el estudio de aceite vegetal como combustible para motores de combustión interna (Snare et al., 2007; Sharma y Singh 2009).

Sin embargo, los aceites vegetales puros no se pueden utilizar como combustibles. Su uso in natura última instancia, puede causar problemas con las boquillas del motor, así como la quema incompleta, lo que lleva a la formación de coque, lo que puede causar daños importantes a medio y largo plazo (Morais, 2010; Guerra y Fuchs, 2010), necesitando la reducción de su viscosidad (Morais, 2010), que por procedimiento químico, convirtiendo el aceite en biodiesel, a través de procesos como el agrietamiento, esterificación y/o transesterificación, que se clasifican como combustibles biodegradables derivados de fuentes renovables (Silva y Freitas, 2008; Castro, 2009).

De acuerdo con la Ley $\mathrm{N}^{\circ} 9478$ de 6 de agosto de 1997 , junto con la Ley $N^{0} 11.097$, de 2005 , se considera biodiesel y biocombustibles a partir de biomasa renovable para su uso en motores de combustión interna de encendido por compresión, o de acuerdo con las regulaciones de generar otra tipo de energía que puede sustituir parcial o totalmente de combustibles fósiles (Brasil, 2005a) que consiste en esteres metílicos de aceites vegetales o grasas animales, que pertenece a la clase de combustible ecológico, debido a su composición cualitativa del $77 \%$ de carbono, $12 \%$ de hidrógeno, $11 \%$ de oxígeno, y trazas de nitrógeno y azufre (Encinar et al., 2005). 
Las mayoría de los estudios relacionados con la producción de biodiesel citan la transesterificación de aceites comestibles tales como colza, soja, girasol, colza, etc., utilizando metanol y $\mathrm{NaOH} / \mathrm{KOH}$ como catalizador (Meher et al., 2006).

Sin embargo, cada vez mas surgen trabajos utilizando como materia prima aceites residuales domésticos (Leung y Guo, 2006; Seamus 2008; Predojević, 2008; Alvarenga y Smith, 2010; Araujo et al., 2013; Guimaraes et al., 2014).

\subsection{Materias primas principales}

Aunque Brasil presentes condiciones de clima y suelo favorables para el cultivo de diferentes variedades de aceite para la producción de biodiesel, sin embargo, la producción se limita a unas pocas materias primas tales como el soja correspondiendo el $75 \%$ de la producción en el año 2014. La grasa animal aparece en segundo, de $20 \%$, seguido de aceite de semilla de algodón $(2 \%)$ y aceite de freír los residuos (1\%). Las otras materias primas representan el $2 \%$ de la producción de biodiesel en 2014 (Tabla 1) (Abiove, 2015a).

Tabla 1.Producción de biodiesel por materia prima $\left(\mathrm{m}^{3}\right)$.

\begin{tabular}{|l|l|l|l|l|l|}
\hline & \multicolumn{1}{|c|}{$\mathbf{2 0 1 0}$} & \multicolumn{1}{|c|}{$\mathbf{2 0 1 1}$} & \multicolumn{1}{|c|}{$\mathbf{2 0 1 2}$} & \multicolumn{1}{c|}{$\mathbf{2 0 1 3}$} & $\mathbf{2 0 1 4}$ \\
\hline Aceite de soja & $1.960 .822,0$ & $2.152 .298,0$ & $2.041 .667,0$ & $2.142 .990,0$ & $2.551 .813,0$ \\
\hline Sebo animal & $330.574,0$ & $367.578,0$ & $481.231,0$ & $611.215,0$ & $731.935,0$ \\
\hline Aceite de algodón & $57.458,0$ & $84.711,0$ & $123.247,0$ & $65.960,0$ & $81.666,0$ \\
\hline Aceite de freír usado & $4.751,0$ & $13.044,0$ & $17.827,0$ & $30.667,0$ & $25.949,0$ \\
\hline Otras & $32.835,0$ & $55.130,0$ & $53.511,0$ & $66.664,0$ & $28.475,0$ \\
\hline Total & & & & & \\
\hline
\end{tabular}

Fuente: (Abiove, 2015a).

El gran uso de aceite de soja se debe a la gran excedente de aceite existente en el mercado, debido a la gran demanda de proteína vegetal, junto con la organización de la cadena de producción (Graef et al., 2010).

Iniciativas son necesarias para la diversificación de cultivos para proporcionar opciones para el cultivo en zonas donde los productos tradicionales como la soja, el trigo, el maíz, entre otros, tienen escaso valor económico y la productividad por unidad de superficie (Vaccaro et al., 2010).

La Tabla 2 muestra el equilibrio de la oferta y la demanda de aceite vegetal de soja para 2008 a 2014. Al parecer, la producción de aceite de soja en 2014 fue de alrededor de 7 mil millones de toneladas, de las cuales $82 \%$ son para consumo interno (Abiove, 2015b).

Tabla 2. Complexo da Soja: Balanço Oferta/Demanda de óleo vegetal (1.000 t).

\begin{tabular}{|l|l|l|l|l|l|l|l|}
\hline \multicolumn{1}{|c|}{ Discriminación } & \multicolumn{1}{|c|}{$\mathbf{2 0 0 8}$} & $\mathbf{2 0 0 9}$ & $\mathbf{2 0 1 0}$ & $\mathbf{2 0 1 1}$ & $\mathbf{2 0 1 2}$ & $\mathbf{2 0 1 3}$ & $\mathbf{2 0 1 4}$ \\
\hline Estoque inicial & 388,0 & 358,0 & 311,0 & 361,0 & 391,0 & $314,0,0$ & 288,0 \\
\hline Producción & $6.267,0$ & $5.896,0$ & $6.928,0$ & $7.340,0$ & $7.013,0$ & $7.075,0$ & $7.200,0$ \\
\hline Importación & 27,0 & 27,0 & 16,0 & 0 & 1,0 & 5,0 & 0 \\
\hline Consumo Doméstico & $4.102,0$ & $4.454,0$ & $5.404,0$ & $5.528,0$ & $5.328,0$ & $5.723,0$ & $5.950,0$ \\
\hline Exportación & $2.222,0$ & $1.517,0$ & $1.490,0$ & $1.782,0$ & $1.764,0$ & $1.383,0$ & $1.250,0$ \\
\hline Estoque Final & 358,0 & 311,0 & 361,0 & 391,0 & 314,0 & 288,0 & 288,0 \\
\hline
\end{tabular}


Según Rabelo y Ferreira (2008), la relación con el uso y eliminación de aceite para freír es del orden del $53 \%$, está claro lo que un potencial de aproximadamente 3153 millones de toneladas de aceite residual que podrían asignarse para la producción de biodiesel.

A pesar de tener potencial para la producción de biodiesel, la grasa bovina, aceites residuales no son materias primas privilegiadas por PNPB y no gozan de los beneficios fiscales del Programa Nacional de Producción y Uso de Biodiesel (PNPB) (Freitas y Lucon, 2011).

\subsection{Proceso de Producción (B100)}

La forma más común para producir el biodiesel es por nivel nacional de transesterificación, que se refiere a una reacción química catalizada con participación de aceite vegetal y un alcohol para obtener esteres alquílicos de ácidos grasos (biodiesel) y glicerina (Zhang et al., 2003).

Triglicéridos aparecen como principal componente aceite vegetal, compuesto por tres moléculas de ácidos grasos unidas a una molécula de glicerol y ácidos grasos libres (AGL). En el proceso de transesterificación, los triglicéridos presentes en el aceite se convierten en esteres de moléculas más pequeñas de ácidos grasos (biodiesel) y glicerina (Zhang et al., 2003).

El proceso de transesterificación se produce usando alcohol primario, tal como metanol, etanol, propanol y butanol en presencia de un catalizador. El metanol y el etanol son los más utilizado debido al bajo costo y la disponibilidad en el mercado. En comparación el rendimiento de los esteres etílicos de ácidos grasos en comparación con los esteres metílicos son más pequeñas y de difícil separación de glicerol, causada por problemas en el proceso etanólisis" (Meher et al., 2006).

En cuanto al uso del catalizador, la reacción más utilizada en la industria es la catálisis básica usando hidróxido de sodio, hidróxido de potasio y metóxido de sodio. Estos catalizadores presentan menor costo e menor tiempo de reacción y una mayor disponibilidad en el mercado (Freedman et al., 1984), permitiendo velocidades de reacción 4.000 veces superiores a los obtenidos con la misma cantidad de catalizadores ácidos (Souza, 2006).

Sin embargo, cuando se utilizan aceites puros o no, con altos niveles de ácidos grasos libres, puede formarse jabones, causando saponificación de triglicéridos, siendo indeseable porque afectan el rendimiento, la separación y purificación de esteres de biodiesel (Maa y Hanna, 1999; Vicente et al., 2004), se deben hacer neutralización alcalina con hidróxido de sodio o de potasio y, posteriormente, de des humidificación (Araujo, 2013; Parente, 2003).

Pero otra opción de producción de biodiesel, cuando la materia prima tiene alto nivel de ácidos grasos libres, es utilizar un catalizador ácido tal como ácido sulfúrico y ácido clorhídrico. Que conduce a altos rendimientos de esteres (99\%), sin embargo, son reacciones lentas, que requieren altas temperaturas (por encima de $100^{\circ}$ C) y los períodos de reacción largos. Pero, sin embargo, la transesterificación ácido es una materia prima excelente para con alta cantidad de ácidos grasos libres (Demirbas, 2009).

La principal ventaja de transesterificación en medios ácidos es la formación de jabón además, los ácidos grasos libres presentes en el aceite reaccionan por esterificación, convirtiéndose en biodiesel, aumentando así el rendimiento de la reacción y facilitar la separación y la purificación de este biodiesel (Parente, 2003; Bondioli, 2004). Otras variables relevantes en el proceso con tal intensidad de agitación (RPM), la temperatura y el tiempo de reacción (Marchetti et al., 2007).

\subsection{El costo de producción (B100)}

Actualmente, el alto costo de producción es el principal obstáculo en la producción nacional de biodiesel, causada principalmente por el alto costo de las materias primas, en la que refleja directamente el coste final (Zhang et al., 2003; Sharma y Singh 2009). Además de la escala de producción y las altas tasas de impuestos insertados en el combustible (Padula et al., 2013).

De acuerdo con la Asociación Brasileña de Industrias de Aceites Vegetales (ABIOVE), el costo de producción se divide en materia prima (aceite vegetal $80 \%$ ), alcoholes (metanol / etanol 10\%) y los costos de operación (10\%) (Abiove, 2006c; Zonin, 2008).

Al parecer, en la figura 4, el precio cobrado por las empresas procesadoras de aceite vegetal (Biomercado), es igual al precio promedio pago en las subastas de la Agência Nacional do Petróleo, Gás Natural e Biocombustíveis (ANP), en promedio R \$1,96 / litro (Abiove, 2015d).

Fioresei et al. (2011), utilizando diferentes materias primas para la producción de biodiesel a pequeña escala, se encontró que el costo final de biodiesel está directamente influenciado con la materia prima, entre 
los materiales evaluados, sebo vacuno mostró el costo más bajo entre las demás ( $\mathrm{R} \$ 2,01$ / litro), seguido por el aceite de pollo ( $R$ \$ 2,22 / I), soja ( $\mathrm{R} \$ 3,23$ / I), girasol (R \$ 3,34 / I).

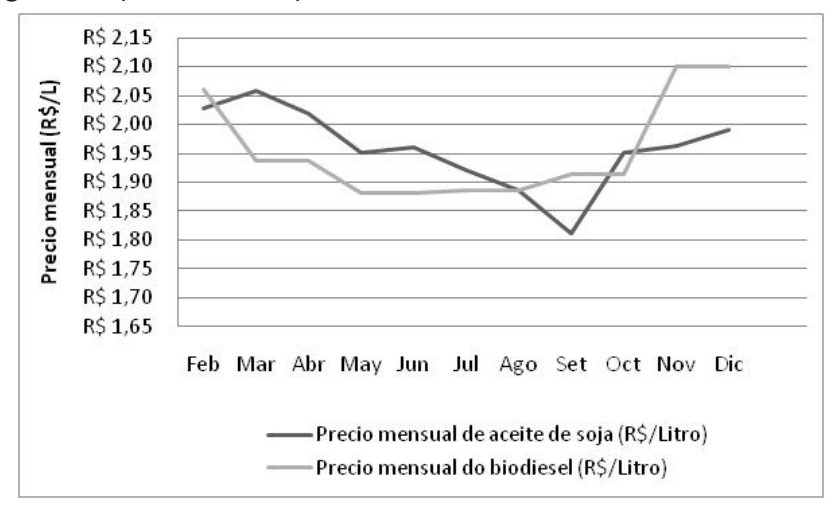

Figura 2. Precio mensual de aceite de soja y biodiesel en 2014 ( $\mathrm{R}$ \$ / litro).

Fuente: (Abiove, 2015d; Biomercado, 2015).

El costo de biodiesel litro es $60 \%$ mayor que el litro de diesel mineral. Además, muchas veces la producción está lejos de los grandes centros de consumo elevando considerablemente los costos de logística (Fecombustíveis, 2012).

\subsection{La producción brasileña de (B100)}

La producción de biodiesel en Brasil se inició en 1975 a través del Plan de Producción Vegetal de aceite con fines energéticos (PROÓLEO), coordinado por el Ministerio de Agricultura. Sin embargo, nunca se llevó a cabo y fue reemplazado por el Programa Nacional del Alcohol (PROALCOOL), tiempo después de fallar por diversas razones, encerrando las actividades (Castellanelli et al., 2006).

Pero con los años, el interés por biodiesel regresó a la escena nacional, en particular mediante el aumento de la población, la disminución de los recursos fósiles, las limitaciones ambientales y el aumento de los precios del petróleo, así como el crecimiento de la producción y el consumo en los países de Europa, y creó el Programa Brasileño de Biodiesel (Pro-biodiesel) por el Decreto n. 720 de 30 de octubre 2002. En 2003, se creó el Programa Nacional de Producción y Uso de Biodiesel (PNPB), definiendo como prioridad la expansión de la producción y el consumo a escala comercial de una manera sostenible, con inclusión social y el desarrollo regional y la diversificación de las materias primas, destinadas a la generación de empleos e ingresos para la población (Mattei, 2010). Otra de las prioridades del programa era introducir y mantener el uso de las energías renovables en la matriz energética brasileña con el fin de reducir la dependencia nacional del petróleo y su cadena de suministro (Vaccaro et al., 2010).

La Figura 2, muestra que a principios de los años en que el (PNPB) fue creado, la producción de biodiesel Nacional de era insignificante con sólo 736.000 litros en 2005. En 2010, después de cinco años de la creación de (PNPB), hubo un crecimiento significativo de la producción, 2.386 mil millones de litros. En 2013, la producción aumentó en un 5,35\% en comparación con 2012 produciendo 2.917 mil millones de litros. En el año 2014 la producción aumentó un 17\% respecto al año anterior siendo producido 3.414 mil millones de litros de biodiesel (ANP, 2015).

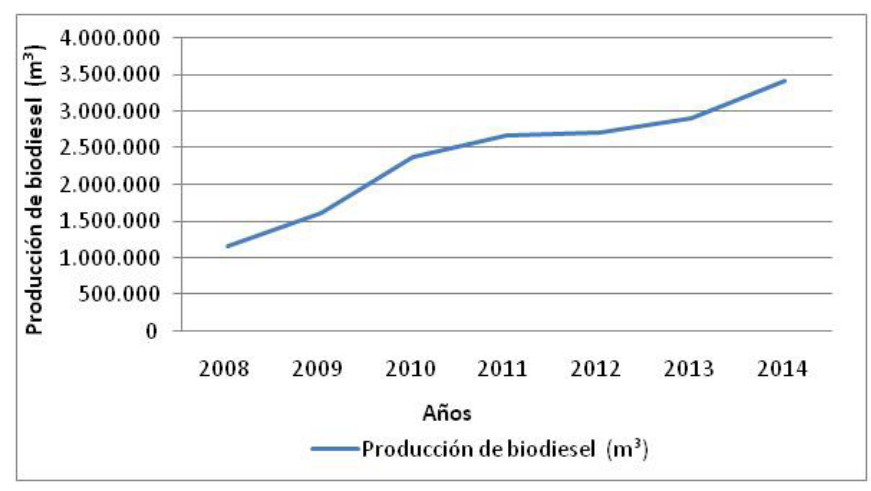

Figura 3. Producción brasileña-B100 $\left(\mathrm{m}^{3}\right)$ entre 2005-2014 Fuente: (ANP, 2015).

Sin embargo, a pesar de una producción creciente anualmente, esta permanece abajo de la capacidad autorizada. En diciembre de 2014, la capacidad autorizada para la operación comercial fue de7.502 millones de litros, lo que demuestra una capacidad ociosa del $46 \%$ en la producción (MME, 2015) .Sobre todo esta capacidad es causada por el reducido número de materias primas para la producción de biodiesel, que se menciona en la sección anterior.

\subsection{La demanda nacional (B100)}

En Brasil, la demanda de biodiesel está vinculada principalmente a las políticas aplicadas por el gobierno a través de los mecanismos legales que determinan la demanda y el volumen de la mezcla con el diesel.

En 2006, el biodiesel ha empezado a ser añadido al diesel y se ha vuelto obligatorio a partir de 2008. Entre enero y junio de 2008 , la mezcla de biodiesel puro (B100) al diesel fue de $2 \%$ a partir de entonces, entre julio de 2008 y junio 2009 3\% al 4\% entre julio y diciembre 2009. En 2010, todo el combustible diesel de automoción vendidos en el país recibió la adición de volumen biodiesel 5\% como Resolución CNPE No. 6 
de 16 de noviembre de 2009 (MME, 2009).

Por el momento, de acuerdo a la Ley 13.033 / 2014 actual, el biodiesel comenzó a ser agregado al combustible diesel a un ritmo de $7 \%$ desde el 01 de noviembre 2014 (Brasil, 2014b), por lo que el sector del transporte consumió 2.364 mil millones de litros de biodiesel en el año de 2013.

Se puedeobservar en la Figura 3, el diesel fue el responsable por $46,4 \%$ de la generación de energía, seguido de gasolina $(29,4 \%)$, etanol $(14,3 \%)$, combustible de aviación (4,3\%) y el 2,3\% para el biodiesel (EPE, 2014).

El transporte por carretera ha absorbido el $97 \%$ (2304 millones de litros) con adición hecha al diesel, y el $3 \%$ por ferrocarril. En los próximos años se espera que esta demanda aumente, impulsada por el aumento de la proporción de la mezcla que se añade al diesel, de acuerdo a la Ley 13.033 / 2014, vigente en el momento (Brasil, 2014). El resto de la producción nacional de biodiesel fue absorbida por sector agropecuario e industrial (14\%) (EPE, 2014).

\section{Consideraciones finales}

Con el objetivo de caracterizar y analizar la situación actual de la producción y la demanda brasileña de biodiesel, al parecer la producción nacional de biodiesel es extremadamente dependiente del aceite de soja como materia prima, en el 2014 representó el $75 \%$ de la producción, con un record de 3.072 mil millones de litros de biodiesel, el $17 \%$ de la producción supero al año de 2013. También, se encontró en el 2013, el $86 \%$ de la producción de biodiesel fue absorbido por el sector del transporte, lo que representa el $2,4 \%$ de consumo total energía. Considerando que el sector de transporte por carretera consume 2.304 mil millones de litros, representando el $97 \%$ del total asignado.

Sin embargo, la importancia del crecimiento de la producción de biodiesel en los últimos años, vienen existiendo varios obstáculos, como: la capacidad ociosa provocadas por la tecnología, infraestructura y agronómicas, además el costo de producción del biodiesel en comparación con el diésel mineral, debido a los altos costos de la materia prima, representando más del $80 \%$ del costo total.

En la actualidad las nuevas investigaciones se están enfocando en el desarrollo de los procesos de producción que utilizan catalizadores enzimáticos. En estos procesos no se requiere la purificación de la materia prima suministrada con alto contenido de ácidos grasos libres que proporcionan un alto rendimiento y menor costo de producción final. También, permiten una mayor utilización de los aceites que se descartan cada día por la población y hasta el momento son poco explorados, representando sólo el $1 \%$ de la producción de biodiesel, en comparación con las otras materias primas.

\section{Referencias}

ABIOVE, Associação Brasileira das Indústrias de Óleos Vegetais. (2015a). Produção de biodiesel por matéria-prima (\%). Disponível em: <http://www.abiove.org.br/>. Acesso em 14 jan. 2015.

ABIOVE, Associação Brasileira das Indústrias de Óleos Vegetais. (2015b). Brasil- Complexo Soja. Disponível em:<http://www.abiove.org.br/>. Acesso em 14 jan. 2015.

ABIOVE, Associação Brasileira das Indústrias de Óleos Vegetais. (2015c). A visão da indústria de óleos vegetais. $6^{\circ}$ Fórum de debates sobre qualidade e uso de combustíveis. IBP. Disponível em:<http:// www.abiove.org.br/>. Acesso em 14 jan. 2015.

ABIOVE, Associação Brasileira das Indústrias de Óleos Vegetais. (2015d). Preços nominais mensais do biodiesel: média dos leilões ANP $\left(R \$ / \mathrm{m}^{3}\right)$. Disponível em:<http://www.abiove.org.br/>. Acesso em 14 jan. 2015.

ALVARENGA, B. M.; SOARES, M. A. (2010). "Potencialidade de produção de biodiesel por óleos e gorduras residuais na cidade de Itabira-MG". Revista Ceres, 57 (6), pp. 721-729.

ANEEL, Agência Nacional de Energia Elétrica. (2008). "Atlas de energia elétrica do Brasil. 3". Disponível em: <http://www.aneel.gov.br/>. Acesso em 14 jan. 2015

ANP, Agência Nacional do Petróleo, Gás Natural e Biocombustíveis. (2015). "Produção de biodiesel B100 por produtor - 2005-2014 (bep)". Disponível em: <http://www.anp.gov.br>. Acesso em: jul. 2015.

BECKER, D. R., MOSELEY, C., LEE, C. (2011). "A supply chain analysis framework for assessing state-level forest biomass utilization policies in the United States". Biomass and bioenergy, 35 (4), pp. 1429-1439.

BIOMERCADO - Centro de Referência da Cadeia de Produção de Biocombustíveis para Agricultura Fa- 
miliar. (2015). Boletim do Complexo soja. Campus Universitário. Viçosa, MG.

BONDIOLI, P. (2004). "The preparation of fatty acid esters by means of catalytic reactions". Topics in Catalysis, 27 (1-4), pp. 77-82.

BRASIL, Casa Civil. (2005a). Subchefia de Asuntos Jurídicos. Ley N 9478 de 6 de agosto de 1997. Brasilia, Brasil.

BRASIL, Casa Civil. (2014b). Subchefia de Asuntos Jurídicos. Lei $N^{\circ} 13.033$, de 24 de setembro de 2014, Brasilia, Brasil.

CASTELLANELLI, M., SOUZA, S. N. M. de, SILVA, S. L., KAILER, E. K. (2008). "Desempenho de motor ciclo Diesel em bancada dinamométrica utilizando misturas diesel/biodiesel". Engenharia. Agrícola, 28 (1), pp. 145-153.

CORONADO, C. R., de CARVALHO, J. A., YOSHIOKA, J. T., SILVEIRA, J. L. (2009). "Determination of ecological efficiency in internal combustion engines: The use of biodiesel". Applied Thermal Engineering, 29 (10), pp. 1887-1892.

DEMIRBAS, A. (2009). "Progress and recent trends in biodiesel fuels". Energy Conversion and Management, 50, p. 14-34.

ENCINAR , J. M., JUAN F. GONZÁLEZ , J. F; RODRÍGUEZ-REINARES, A. (2005). "Biodiesel from used frying oil. Variables affecting the yields and characteristics of the biodiesel". Industrial \& Engineering Chemistry Research, 44 (15), pp. 54915499.

EPE, Empresa de Pesquisa Energética (2014). Balanço Energético Nacional 2014. Ano base 2013: Relatório Síntese Rio de Janeiro: EPE, p. 54. Disponível em: <https://ben.epe.gov.br/>. Acesso em: 20 fev. 2015.

FERREIRA, V. F., ROCHA, D. R. DA, SILVA, F. C. da. (2014). "Química Verde, Economia Sustentável e Qualidade de Vida”. Revista Virtual Química, 6 (1), pp. 85-111.

FIORESEI, D. A., GOMES, L. F. S., SOUZA, SAMUEL N. M. DE; DALLMEYERI, A. U., ROMANO, L. N. (2011). "Metodologia experimental para avaliação de custos de produção e utilização de biodiesel: estudo de caso de quatro ésteres metílicos e óleo diesel comercial". Ciência Rural, 41(11), pp.1921-1926.
FOSCHIERA, I. P. (2008). O programa nacional de produção e uso de biodiesel: Impactos e perspectivas. Porto Alegre, $58 \mathrm{f}$.

FREEDMAN, B., PRYDE, E.H., MOUNTS, T.L. (1984). "Variables affecting the yields of fatty esters from transesterified vegetable oils". Journal of the American Oil Chemists Society, 61 (10), pp. 1638-1643.

FREITAS, S.; LUCON, O. (2011). Programa Nacional de Produção e Uso de Biodiesel: a transição para um estilo de desenvolvimento sustentável. Textos para Discussão, n. 27.

GIL, A. C. (1994). Métodos e técnicas de pesquisa social. 4 ed., São Paulo: Atlas.

GRAEF, N. et al. (2010). "O mercado do biodiesel e o ambiente institucional”. In: Encontroparanaense de economia (Ecopar), Anais. Maringá.

GUERRA, E. P., FUCHS, W. (2010). "Biocombustível renovável: uso de óleo vegetal em motores". Revista Acadêmica Ciência Agrária Ambiental, Curitiba, 8 (1), p. 103-112.

GUIMARÃES, R., LOBO, B. B., NUNES, D., AMARAL, E. C. do; MORAES, A. C. de. (2014). "Avaliação de tipos de pré-tratamento de óleo usado de fritura para produção de biodiesel". Revista de Extensão e Iniciação Científica SOCIESC-REIS, 1 (1).

HAMMOND, G., KALLU, S., MCMANUS, M. (2008). "Development of biofuels for the UK automotive market”. Applied Energy, 85 (6), pp. 506-51.

LEUNG, Y., GUO, Y. (2006). "Transesterification of neat and used frying oil: Optimization for biodiesel production". Fuel Processing Technology, 87 (10), pp. 883-890, 2006.

LIMA, P. C. R. (2005). Biodiesel: um novo combustível para o Brasil. Consultoria Legislativa da Câmara dos Deputados, 2005.

MAA, F., HANNA, M. A. (1999). "Biodiesel production: a review". Bioresource Technology, 70 (1), pp.1-15.

MARCHETTI, J. M., MIGUEL, V. U., ERRAZU, A. F. (2007). "Posibles métodos para la producción de biodiesel". Renovables y Sostenibles Energy Reviews, 11, pp. 1300-1311.

MARTINS, H. H. T. de S. (2004). Metodologia qualitativa de pesquisa. Educação e Pesquisa, São Paulo, 30 (2), pp. 289-300. 
MATSUMOTO, N., SANO, D., ELDER, M. (2009). "Biofuel initiatives in Japan: strategies, policies, and future potential". Applied Energy, 86, pp. S69-S76

MATTEI, L. F. (2010). "Programa Nacional para Produção e Uso do Biodiesel no Brasil (PNPB): trajetória, situação atual e desafios". Revista Econômica do Nordeste, Fortaleza, 41 (4), p. 731-740.

MEHER, L. C., VIDYA, S. D., NAIK, N. S. (2006). "Technical Aspects of Biodiesel production by the transesterification-review". Renewable and Sustainable Energy Reviews, 10, pp. 248-268.

MELLO, M. G. (2001). Biomassa: Energia dos trópicos em minas gerais. Labmídia. Belo Horizonte.

MINAYO, M. C. de S. (2003). "Ciência, técnica e arte: o desafio da pesquisa social". In: MINAYO, M. C. de S. (Org.). Pesquisa social: teoria, método e criatividade. 22. ed. Petrópolis: Vozes, pp. 9-30.

MME, Ministério de Minas e Energia (2009). Resolução CNPE n 6, de 16.9.2009 - DOU 26.10.2009. Brasil.

MORAIS, P de. (2010). "Tecnologias em uso e disponíveis no exterior e no Brasil para o uso de óleo vegetal refinado como combustível". In: FARIA, et al. A Utilização de Óleo Vegetal Refinado como Combustível - Aspectos Legais, Técnicos, Econômicos, Ambientais e Tributários. Textos para Discussão 73.

PADULA, A. D.; SANTOS, M. SILVEIRA; FERREIRA, L.; BORENSTEIN, D. (2012). "The emergenceof the biodiesel industryin Brazil: Currentfigures and future prospects". Energy Policy, 44, pp. 395-405.

PARENTE, E. J. de Sá. (2003). Biodiesel: Uma Aventura Tecnológica num País Engraçado.

PREDOJEVIC, Z. J. (2008). "The production of biodiesel from waste frying oils: A comparison of different purification steps". Fuel, 87 (17), pp. 3522-3528.

PUPPÁN, D. (2002). "Environmental evaluation of biofuels". Periodica Polytechnica Social and Management Sciences, 10(1), pp. 95-116.

RABELO, R. A., FERREIRA, O. M. (2008). Coleta seletiva de óleo residual de fritura para aproveitamento industrial. Ambiente em Foco. Departamento de Engenharia-Engenharia Ambiental, Universidade Católica de Goiás.
REI, P. A. G. V. (2007). Procedimentos laboratoriais para o controlo da qualidade do Biodiesel. Lisboa. Universidade Técnica Lisboa. Lisboa-Portugal.

SANTOS, F. A. (2010). Biocombustíveis: prós e contras.

SHARMA, Y. C., SINGH, N. B. (2009). "Development of biodiesel: current scenario". Renewable and Sustainable Energy Reviews, 13 (6), pp. 1646-1651.

SILVA, P. R. F. da, FREITAS, T. F. S. de. (2008). "Biodiesel: o ônus e o bônus de produzir combustível". Ciência Rural, 38 (3), pp. 843-851.

SINGH, P. S. and SINGH D. (2010). "Biodiesel production through theuseof different sources and characterization of oils and Their esters of the substitute of diesel: A review". Renewable and Sustainable Energy Reviews, 14 (1), pp. 200-216.

SNÅRE, M., KUBIČKOVÁ, I., MÄKI-ARVELA, P., ERÄNEN, K., WÄRNÅ, J., \& MURZIN, D. Y. (2007). "Production of diesel fuel from renewable feeds: kinetics of ethyl stearate decarboxylation". Chemical Engineering Journal, 134 (1), pp. 29-34.

SOUZA, C. A. (2006). Sistemas catalíticos na produção de biodiesel por meio de óleo residual. Ano 6 . Encontro de Energia Meio Rural.

TRIVIÑOS, A. N. S. (1987). Introducción a la investigación en ciencias sociales. São Paulo: Atlas, pp. 175.

TSAI, W-T. (2009). "Coupling of energy and agricultural policies on promoting the production of biomass energy from energy crops and grasses in Taiwan". Renewable and Sustainable Energy Reviews, 13 (6), pp. 1495-1503.

VACCARO, G. L. R. et al. (2010). "Escenarios futuros para la cadena de biodiesel del estado brasileño". Los comentarios de Energía Renovable y Sostenible, 14, pp. 1263-1272.

VICENTE, G. (2001). Study of the biodiesel production. PhD Thesis, Faculty of Chemistry. Complutense University of Madrid.

VICENTE, G., MARTINEZ, M., ARACIL, J. (2004). "Integrated biodiesel production: a comparison of different homogeneous catalysts systems". Bioresource technology, 92 (3), pp. 297-305. 
VIEIRA, P. R. C. (2014). Análise comparativa de investimentos de processo produtivos de biodiesel com auxílio de uma planilha eletrônica.Recife. Universidade Católica de Pernambuco, Recife.

ZHANG, Y., DUBE, M. A., MCLEAN, D. D., KATES, M. (2003). "Biodiesel production from waste cooking oil: 2. Economicassessmentandsensiti-vityanalysis". Bioresource technology, 90 (3), pp. 229-240.
ZONIN, V. (2008). Potenciais e Limitações da Indústria de Biodiesel no Brasil: um Estudo de Caso. Universidade do Vale do Rio dos Sinos, São Leopoldo. 